Theoretical study of the electronic states of hollandite vanadate $\mathrm{K}_{2} \mathrm{~V}_{8} \mathrm{O}_{16}$

This content has been downloaded from IOPscience. Please scroll down to see the full text. 2009 J. Phys.: Conf. Ser. 150042182

(http://iopscience.iop.org/1742-6596/150/4/042182)

View the table of contents for this issue, or go to the journal homepage for more

Download details:

IP Address: 194.95.157.184

This content was downloaded on 05/05/2017 at 08:58

Please note that terms and conditions apply.

You may also be interested in:

Oxidization/Reduction Process of Prussian Blue Film as Investigated by Valence-Differential Spectroscopy

Yutaro Kurihara, Hayato Kamioka, Fumiya Nakada et al.

Roles of Electron Hopping on Orbital Ordering for Vanadium Spinels

Huang Yuan-Jie and Zhang Yu-Heng

Magnetic interactions and orbital state in double chain systems investigated by NMR measurements Masayuki Itoh, Hikaru Takeda, Yasuhiro Shimizu et al.

$\underline{\text { Anomalous electronic states of hollandite-type transition-metal oxides }}$

Y Ohta, T Toriyama, M Sakamaki et al.

Magnetic, structural, and thermal properties of CoV2O4

Yuanjie Huang, Zhaorong Yang and Yuheng Zhang

Ac susceptibility and 51V NMR study of MnV2O4

S-H Baek, K-Y Choi, A P Reyes et al. 


\title{
Theoretical Study of the Electronic States of Hollandite Vanadate $\mathbf{K}_{2} \mathbf{V}_{8} \mathbf{O}_{16}$
}

\author{
S Horiuchi ${ }^{1}$, T Shirakawa ${ }^{1,2}$ and Y Ohta ${ }^{1}$ \\ ${ }^{1}$ Department of Physics, Chiba University, Chiba 263-8522, Japan \\ ${ }^{2}$ Institut für Theoretische Physik, Leibnitz Universität Hannover, D-30167 Hannover, \\ Germany
}

\begin{abstract}
Electronic states of hollandite vanadate $\mathrm{K}_{2} \mathrm{~V}_{8} \mathrm{O}_{16}$, a one-dimensional zigzag-chain system of $t_{2 g}$ orbitals in a mixed valent state, are considered. We calculate the Madelung energies to determine the most stable charge-ordering pattern that is consistent with the observed superlattice structure. We then develop the strong-coupling perturbation theory to derive the effective spin-orbit Hamiltonian, starting from the triply-degenerate $t_{2 g}$ orbitals in the $\mathrm{VO}_{6}$ octahedral structure. An exact-diagonalization technique is used on small clusters of this Hamiltonian to determine the orbital-ordering pattern and spin structures in the ground state. We thereby discuss the electronic and magnetic properties of $\mathrm{K}_{2} \mathrm{~V}_{8} \mathrm{O}_{16}$.
\end{abstract}

Recently, it has been reported [1] that a hollandite vanadate compound $\mathrm{K}_{2} \mathrm{~V}_{8} \mathrm{O}_{16}$ shows a metal-insulator transition (MIT) at $\sim 170 \mathrm{~K}$, which is accompanied by the rapid reduction of the magnetic susceptibility. Below the transition temperature, a characteristic superlattice of $\sqrt{2} a \times \sqrt{2} a \times 2 c$ is observed [1], whereby a possible CO phase transition accompanied by the spin-singlet formation has been proposed [1]. The crystal structure of this compound belongs to a group of hollandite-type phase and has a $\mathrm{V}_{8} \mathrm{O}_{16}$ framework composed of double strings of edge-shared $\mathrm{VO}_{6}$ octahedra. Our system $\mathrm{K}_{2} \mathrm{~V}_{8} \mathrm{O}_{16}$ has the average valence of $\mathrm{V}^{3.75+}$ and thus is in the mixed valent state of $\mathrm{V}^{3+}: \mathrm{V}^{4+}=3 d^{2}: 3 d^{1}=1: 3$. Thus, the central issue in the present system is the mechanism of the MIT concerning how the highly frustrated spin, charge, and orbital degrees of freedom at high temperatures are relaxed by lowering temperatures and what type of orders is realized in the ground state.

In this paper, we first calculate the Madelung energy and obtain several $\mathrm{CO}$ patterns and their relative stability to determine the most stable one that is consistent with the characteristic superlattice structure observed in the low-temperature phase of the material. We then develop the strong-coupling perturbation theory starting from the triply-degenerate $t_{2 g}$ orbitals in the $\mathrm{VO}_{6}$ octahedral structure and derive the effective spin-orbit Hamiltonian in the approximation of neglecting the orbital fluctuations. We apply an exact-diagonalization technique on small clusters to this Hamiltonian and obtain the orbital-ordering (OO) pattern and spin structures in the ground state. We thereby suggest that the state of local singlets of two $s=1 / 2$ spins coexisting with local high-spin clusters of $S=3 / 2$ should be of possible relevance with the observed electronic and magnetic states of $\mathrm{K}_{2} \mathrm{~V}_{8} \mathrm{O}_{16}[2]$.

To see the relative stability of several $\mathrm{CO}$ patterns, we assume various $d$-electron distributions on the $\mathrm{V}$ ions and compare the Madelung energies [3] where we use the room-temperature crystal structure reported for $\mathrm{K}_{2} \mathrm{~V}_{8} \mathrm{O}_{16}$ [4]. We find that a number of $\mathrm{CO}$ patterns are more stable than uniform distribution of electrons on the $\mathrm{V}$ ions. The most stable $\mathrm{CO}$ pattern that is consistent 
with the superlattice structure observed in low-temperature phase of the material is illustrated in Fig. 1. Other calculated CO patterns and their relative stability are discussed in Refs. [2] and $[5]$.

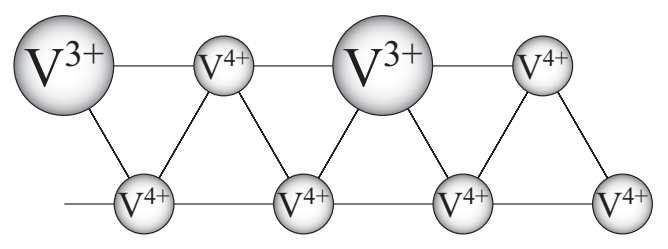

Figure 1. Most stable $\mathrm{CO}$ pattern in $\mathrm{K}_{2} \mathrm{~V}_{8} \mathrm{O}_{16}$, where only a double string of $\mathrm{V}$ ions in the material is illustrated.

We then develop the strong-coupling perturbation theory starting from the ground state in the strong-coupling limit, i.e., the CO state shown in Fig. 1. Our starting high-energy Hamiltonian is of the following form:

$$
\begin{aligned}
H= & H_{0}+H_{t} \\
H_{0}= & V \sum_{\langle i j\rangle} n_{i} n_{j}+V^{\prime} \sum_{[i, j]} n_{i} n_{j}-J_{\mathrm{H}} \sum_{i \sigma \sigma^{\prime}, \alpha \neq \beta} c_{i \alpha \sigma}^{\dagger} c_{i \beta \sigma^{\prime}}^{\dagger} c_{i \beta \sigma} c_{i \alpha \sigma^{\prime}} \\
& +U \sum_{i \alpha} n_{i \alpha \uparrow} n_{i \alpha \downarrow}+U^{\prime} \sum_{i, \alpha \neq \beta} n_{i \alpha} n_{i \beta} \\
H_{t}= & -\sum_{\langle i \alpha, j \beta\rangle, \sigma} t_{i \alpha, j \beta}\left(c_{i \alpha \sigma}^{\dagger} c_{j \beta \sigma}+\text { H.c. }\right)
\end{aligned}
$$

where $c_{i \alpha \sigma}^{\dagger}\left(c_{i \alpha \sigma}\right)$ is the creation (annihilation) operator of an electron at site $i$, orbital $\alpha$, and $\operatorname{spin} \sigma(=\uparrow, \downarrow)$. We define the number operators $n_{i \alpha \sigma}=c_{i \alpha \sigma}^{\dagger} c_{i \alpha \sigma}, n_{i \alpha}=n_{i \alpha \uparrow}+n_{i \alpha \downarrow}$, and $n_{i}=\sum_{\alpha} n_{i \alpha} . V$ and $V^{\prime}$ are the intersite Coulomb repulsions between nearest-neighbor and next-nearest-neighbor sites, respectively. $J_{\mathrm{H}}$ is the Hund's rule coupling, and $U$ and $U^{\prime}$ are the intra- and inter-orbital on-site Coulomb repulsions, respectively. We assume the relation $U^{\prime}=U-2 J_{\mathrm{H}}$ which is valid in the atomic limit. $t_{i \alpha, j \beta}$ is the hopping parameter between the orbital $\alpha$ on site $i$ and orbital $\beta$ on site $j$ where $\alpha, \beta \in\left\{d_{x y}, d_{y z}, d_{z x}\right\}$. We retain only the direct $\mathrm{V}-\mathrm{V}$ hoppings between the $t_{2 g}$ orbitals because the indirect hoppings via the $\mathrm{O}$ ions are rather small [6]. We then have the independent nearest-neighbor hopping parameters $t_{a}, t_{b}$, and $t_{c}$ as shown in Fig. 2.

We assume that the ground state of our Hamiltonian $H_{0}$ should have the CO pattern shown in Fig. 1, where there are no doubly occupied orbitals and no $\mathrm{V}^{2+}$ or $\mathrm{V}^{5+}$ ionic states since these states are highly unrealistic in this material. We therefore impose the condition $U^{\prime}-2 V-J_{\mathrm{H}}>0$.

To consider the real material, we should take into account the effect of distortion of the $\mathrm{VO}_{6}$ octahedra more carefully because the degeneracy of the $t_{2 g}$ orbitals can be lifted. To evaluate the effect of distortion, we calculate the local symmetry of the Madelung site-potential $\phi$ in the point charge model for the real material. Calculated result shows that the orbital $d_{x y}$ (see Fig. 2) is much less stable for the electron to sit on than the other orbitals $d_{y z}$ and $d_{z x}$ are; i.e. $\phi_{x y}>\phi_{y z}=\phi_{z x}$. We therefore assume that the electrons do not occupy the $d_{x y}$ orbital in the ground state as well as in the perturbation processes. A recent NMR experiment [7] seems to support this assumption.

We then develop the second-order perturbation calculation with respect to $t_{i \alpha, j \beta}$ assuming that $t_{i \alpha, j \beta}$ is much smaller than $U, U^{\prime}, V$, and $V^{\prime}$. We thereby derive the effective spin-orbit Hamiltonian. We introduce an approximation here; because the hopping parameters $t_{i \alpha, j \beta}$ take 


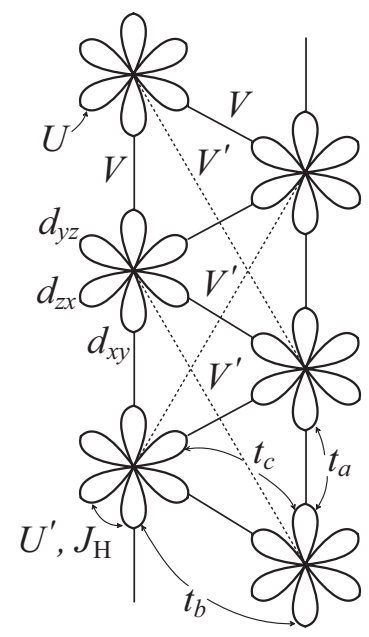

Figure 2. Schematic representation of the $t_{2 g}$ orbitals on the double string of $\mathrm{V}$ ions. Two of the four lobes for each of the three $t_{2 g}$ orbitals are drawn.

the values $t_{a} \gg t_{b} \simeq t_{c}$, we assume $t_{b}=t_{c}=0$ for simplicity as in Refs. [6] and [8]. Note that the orbital fluctuations are completely suppressed in this approximation because only two orbitals connected with the diagonal hopping $t_{a}$ come out and no off-diagonal hopping terms appear in the effective Hamiltonian. We thus obtain the effective spin-orbit Hamiltonian consisting of orbital-diagonal spin-subblocks with vanishing orbital off-diagonal blocks. Complete expression of the effective spin-orbit Hamiltonian is quite lengthy and is presented in Ref. [2].

We calculate the orbital and spin structures in the ground state of the effective spin-orbit Hamiltonian by using the exact-diagonalization technique on small clusters, whereby we discuss its electronic and magnetic properties and compare them with experiment. Hereafter we assume the relation $V^{\prime}=0.6 \mathrm{~V}$ estimated from the experimental interatomic distances between $\mathrm{V}$ ions. We find from the calculated results that the unique orbital state with the total-spin quantum number $S_{\text {tot }}=0$ is realized in the entire parameter space unless the CO state is unstable. We also find from the calculation of the spin-spin correlation functions that the spin structure is quite different in the different regions of the parameter space although the unique OO pattern is stabilized. In the region $J_{\mathrm{H}} \gg V$, we find the results that can roughly be described by the situation where the local high-spin clusters of $S=5 / 2$ formed by four $V$ ions are coupled antiferromagnetically. This state is illustrated schematically in Fig. 3 (a). In the region $V \gg J_{\mathrm{H}}$, we find the results that can be regarded as the state of the local spin singlets of two $s=1 / 2$ spins coexisting with the antiferromagnetically fluctuating local high-spin clusters of $S=3 / 2$. This state is illustrated schematically in Fig. 3 (b). We argue that the latter state can be consistent with available experimental data but the detailed discussions will be presented elsewhere [9].

In summary, we have studied the electronic and magnetic properties in hollandite vanadate $\mathrm{K}_{2} \mathrm{~V}_{8} \mathrm{O}_{16}$, a possible charge and orbital ordering system with the mixed valent state of $\mathrm{V}$ ions with $3 d^{2}: 3 d^{1}=1: 3$ and with the $t_{2 g}$ orbitals of $\mathrm{V}$ ions aligned on the $1 \mathrm{D}$ zigzag chains. First, we have calculated the Madelung energy of the system and obtained the most stable CO pattern that is consistent with the superlattice structure observed in experiment. Then, by using the second-order perturbation theory starting from the triply-degenerate $t_{2 g}$ orbitals in the $\mathrm{VO}_{6}$ octahedral structure, we have derived the effective spin-orbit Hamiltonian. Here, we have evaluated the effect of distortions of the $\mathrm{VO}_{6}$ octahedra from the local symmetry of the Madelung site potential, which justifies the assumption that the electrons do not occupy the $d_{x y}$ orbital. Within the approximation of neglecting the small off-diagonal hopping parameters, we 
(a)

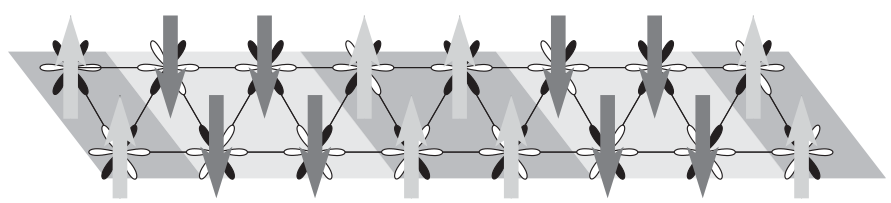

(b)

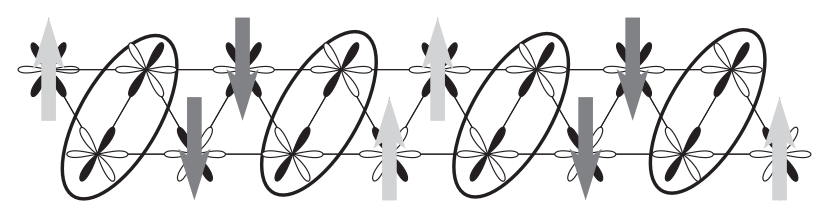

Figure 3. Schematic representation of the orbital and spin structures. Electrons are located in the shaded lobes. (a) The state of the antiferromagnetically fluctuating local high-spin clusters. (b) The state of the local spin-singlets of two $s=1 / 2$ spins (indicated by solid circles) coexisting with the local high-spin clusters of $S=3 / 2$.

have found that the Hamiltonian is block-diagonal with vanishing orbital off-diagonal sectors. We then have used the numerical exact-diagonalization technique on small clusters and have obtained the orbital-ordering pattern and spin structures in the ground state. We thereby have found that depending on the parameter values, either the state of the antiferromagnetically fluctuating local high-spin clusters or the state of the local spin-singlets of two $s=1 / 2$ spins coexisting with the local high-spin clusters of $S=3 / 2$ is realized. By comparing these results with available experimental data which are quite limited at present, we suggest that the latter state can be in agreement with the electronic ground state of hollandite vanadate $\mathrm{K}_{2} \mathrm{~V}_{8} \mathrm{O}_{16}$. We have also performed the first-principles band-structure calculation on this system, of which the results will be published elsewhere [9].

\section{Acknowledgments}

We would like to thank M. Isobe, M. Itoh, and K. Okai for useful discussions on the experimental aspects of $\mathrm{K}_{2} \mathrm{~V}_{8} \mathrm{O}_{16}$. This work was supported in part by Grants-in-Aid for Scientific Research (Nos. 18028008, 18043006, 18540338, and 19014004) from the Ministry of Education, Culture, Sports, Science and Technology of Japan. TS acknowledges financial support from JSPS Research Fellowship for Young Scientists. A part of computations was carried out at the Research Center for Computational Science, Okazaki Research Facilities, and the Institute for Solid State Physics, University of Tokyo.

\section{References}

[1] Isobe M, Koishi S, Kouno N, Yamaura J, Yamauchi T, Ueda H, Gotou H, Yagi T and Ueda Y 2006 J. Phys. Soc. Jpn. 7573801

[2] Horiuchi S, Shirakawa T and Ohta Y 2008 Phys. Rev. B 77155120

[3] See, e.g., Ziman J M 1972 Principles of the Theory of Solids 2nd ed. (London: Cambridge University Press) pp. 37-42.

[4] Abriel W, Rau F and Range K J 1979 Mater. Res. Bull. 141463

[5] Horiuchi S 2008 Master thesis, Chiba University

[6] Pen H F, van den Brink J, Khomskii D I and Sawatzky G A 1997 Phys. Rev. Lett. 781323

[7] Okai $\mathrm{K}$ and Itoh M private communication.

[8] Shibata Y and Ohta Y 2002 J. Phys. Soc. Jpn. 71513

[9] Sakamaki M, Horiuchi S, Konishi T and Ohta Y 2008 in preparation 